\title{
EFFECT OF ELECTROLYTE PROPERTIES ON CURRENT EFFICIENCY OF BIPOLAR PACKED BED ELECTRODES
}

\author{
KATSUKI KUSAKABE, TAKESHI KIMURA, SHIGEHARU MOROOKA \\ AND YASUO KATO \\ Department of Applied Chemistry, Kyushu University, Fukuoka 812
}

Key Words: Electrolysis, Waste Water Treatment, Three Dimensional Electrodes, Current Efficiency, Electrowinning

\begin{abstract}
Electric current densities via different pathways and current efficiencies were evaluated in a bipolar packed bed electrode cell of $0.1 \mathrm{~m} \times 0.1 \mathrm{~m}$ in cross section and $0.2 \mathrm{~m}$ in height. The particulate electrodes were cylindrical ferrite pellets $6 \mathrm{~mm}$ in diameter and $6 \mathrm{~mm}$ long.

The bypass current density was dependent on electrical conductivity of electrolyte, solid holdup, cell configuration and cell voltage. The Faradaic current density under the mass transfer-controlling condition was simulated with the mass transfer coefficient in packed bed, the ratio of effective area to surface area of each pellet, solid holdup and depolarizer concentration. The overall current efficiency was expressed as a function of bypass current density, Faradaic current density and actual current efficiency. Introduction of inert gas bubbles increased the maximum current efficiency by about $15 \%$.
\end{abstract}

\section{Introduction}

In recent years, bipolar particulate electrode cells have attracted much attention in various fields of electrochemistry. ${ }^{2,8,12)}$ When electric potential is applied across the bipolar particulate electrode cell, every electrode particle is polarized and behaves as an anode on one side and a cathode on the other side. Thus, Faradaic reactions take place on both sides of the electrode particle. ${ }^{5)}$ Electrolysis with bipolar particles can be performed in the modes of packed bed, ${ }^{6)}$ fluidized bed ${ }^{9)}$ and trickle bed. ${ }^{4)}$

As shown in the previous paper, ${ }^{10)}$ electric current flowing in the bipolar packed bed is divided into three parts: Faradaic current, bypass current and shortcircuiting current. When the bipolar packed bed cell is applied to the treatment of waste water with a high electrical conductivity, however, the current efficiency decreases remarkably. This seems to be caused by the increase of bypass current in the electrolyte phase, but no evidence has been given to date.

The objective of this work is to clarify the effect of electrical conductivity in the electrolyte phase on the current efficiency of the bipolar packed bed electrode cell. Currents flowing through the different pathways are evaluated by direct measurement. Further, the electrolysis efficiency is improved by introducing nitrogen gas into the bed.

\footnotetext{
Received August 22, 1983. Correspondence concerning this article should be addressed to Y. Kato.
}

\section{Experimental Apparatus and Procedure}

\subsection{Experimental apparatus and electrolyte systems}

The bipolar packed bed electrode cell, shown in Fig. 1, was made of transparent PMMA plastic. The dimensions of the cell were $0.1 \mathrm{~m} \times 0.1 \mathrm{~m}$ in crosssection and $0.2 \mathrm{~m}$ in height. Feeder electrodes were platinum-coated titanium plates of $0.1 \mathrm{~m} \times 0.1 \mathrm{~m}$, and were fixed on opposite walls. The lower edge of the plates was located $5 \mathrm{~cm}$ above the distributor.

The bipolar packed bed consisted of cylindrical sintered ferrite pellets. Each pellet was $6 \mathrm{~mm}$ in diameter and $6 \mathrm{~mm}$ long. These pellets were packed randomly between the two plate electrodes. The solid holdup, $\varepsilon_{s}$, was 0.60 . Glass beads, $12 \mathrm{~mm}$ in diameter, were packed on and beneath the bed of ferrite pellets. The composite pellet shown in Fig. 1 was used to measure the Faradaic current. The structure of the composite pellet was described in the previous paper. ${ }^{10)}$

The electrolyte phase was copper sulphate solutions of $0.01-0.08 \mathrm{~mol} \cdot \mathrm{l}^{-1}$. To adjust the electrical conductivity, sodium sulphate was dissolved in the solutions. The conductivity of the electrolyte was measured with a Kohlrausch bridge. The data are shown in Table 1. The electrolyte was kept at $298 \mathrm{~K}$ and flowed upward in the cell. The superficial liquid velocity was fixed at $0.83 \mathrm{~cm} \cdot \mathrm{s}^{-1}$. The gas phase was a high-purity nitrogen gas.

\subsection{Procedure}

The electrolyte was introduced into the bed and a 


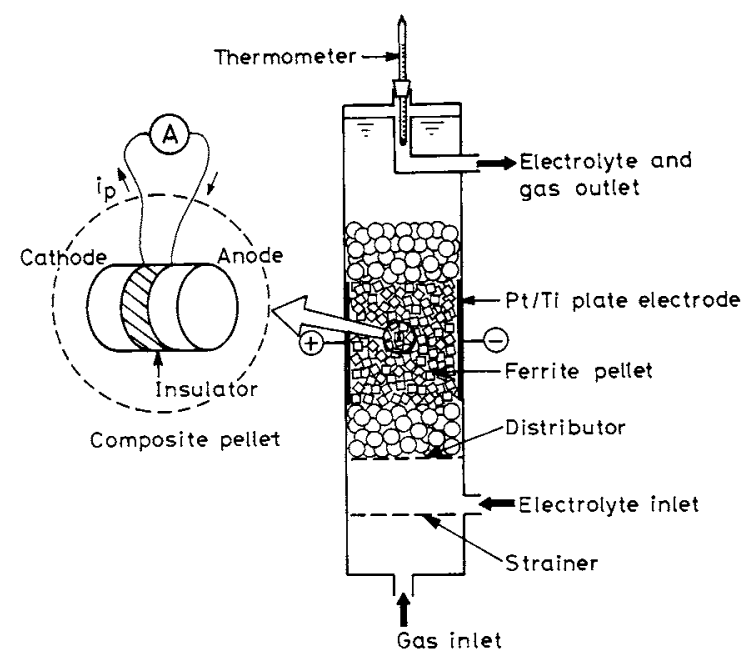

Fig. 1. Cross section of rectangular electrolysis cell.

Table 1. Electrical conductivity of electrolyte

\begin{tabular}{cccc}
\hline $\begin{array}{c}\mathrm{CuSO}_{4} \\
{\left[\mathrm{~mol} \cdot 1^{-1}\right]}\end{array}$ & $\begin{array}{c}\mathrm{Na}_{2} \mathrm{SO}_{4} \\
{\left[\mathrm{~mol} \cdot 1^{-1}\right]}\end{array}$ & $\begin{array}{c}\text { Conductivity } \\
{\left[\mathrm{S} \cdot \mathrm{m}^{-1}\right]}\end{array}$ & Key in Fig. 6 \\
\hline 0.01 & 0 & 0.159 & $\bigcirc$ \\
& 0.02 & 0.469 & $\bigcirc$ \\
& 0.04 & 0.831 & $\mathbf{\bigcirc}$ \\
0.02 & 0.07 & 1.21 & $\bullet$ \\
& 0 & 0.232 & $\triangle$ \\
0.04 & 0.02 & 0.581 & $\mathbf{\Delta}$ \\
0.08 & 0.04 & 0.893 & $\square$ \\
\hline
\end{tabular}

voltage was applied between the two plate electrodes with a stabilized dc power source. The total current flowing through the bed and the Faradaic current flowing through the composite pellet was measured at the same time. The bypass current was measured with inert plastic pellets in place of the ferrite pellets. The size of the plastic pellets was same as the ferrite pellets. The short-circuiting current was measured by using the ferrite bed without electrolyte.

To obtain the current efficiency for copper deposition, the electrolyte was sampled at regular intervals. The concentration of copper ion was determined by chelate titration with EDTA as the titrant and PAN as the indicator.

\section{Results and Discussion}

\subsection{Current density}

The packed bed electrodes are schematically expressed by a three-dimensional stack model of regular cubic unit cells. Each unit cell contains one pellet whose volume is $(\pi / 4) d^{3}$, and the number of unit cells lined up per unit length, $n_{p}$, is given by

$$
n_{p}=(4 / \pi)^{1 / 3} \varepsilon_{s}^{1 / 3} / d
$$

where $\varepsilon_{s}$ is the solid holdup, and $d$ is the size of the pellet. The number of pathways of the Faradaic current in the bed is approximately equal to the number of unit cells placed on the plate electrode. Thus the Faradaic current density based on the area of the plate electrode is given as follows.

$$
j_{p}=i_{p} n_{p}^{2}
$$

where $i_{p}$ is the Faradaic current flowing through a single pellet, and is detected with the composite pellet.

Figure 2 shows typical data of the total current density, $j_{t}$, the bypass current density, $j_{b}$, the Faradaic current density, $j_{p}$, and the sum of $j_{b}$ and $j_{p}$. The current densities are based on the area of the plate electrode. The short-circuiting current was negligible, because the amount of deposited copper was very small in this period. In the range of lower cell voltage, $j_{t}$ was nearly equal to $j_{b}$. When the cell voltage became sufficient for the deposition of copper, the Faradaic current began to flow and copper deposition was observed on the cathodic side of each pellet. In the whole range of cell voltage, $j_{t}$ agreed with the sum of $j_{b}$ and $j_{p}$. The additivity of the currents indicates that the interaction between $j_{b}$ and $j_{p}$ can be neglected.

Figure 3 shows $j_{b}$ and $j_{p}$ for various electrolytes at the cell voltage of $30 \mathrm{~V}$. The bypass current density through the electrolyte phase was proportional to the conductivity of the electrolyte, $\kappa$, and was given by a modification of Bruggemann's equation. ${ }^{3)}$

$$
j_{b}=\alpha \kappa\left(1-\varepsilon_{s}\right)^{1.5} E / L
$$

where $\alpha$ is the cell constant, $E$ is the cell voltage and $L$ is the distance between two plate electrodes. The solid lines of $j_{b}$ in Fig. 3 are calculated from Eq. (3) with $\alpha=1.46$ and 1.0. The cell constant exceeded unity because current pathlines expanded outward from the electrode bed space between the two plate electrodes. When the expansion of current pathlines was avoided by the isolation of the electrode bed space, the cell constant became unity:

On the other hand, the Faradaic current density was affected by the conductivity and the copper concentration. If the reaction of copper deposition is completely controlled by the mass transfer of copper ions, the Faradaic current flowing through a single pellet is expressed as follows.

$$
i_{p m}=(3 / 2) \pi d^{2} f n F k c
$$

where $k$ is the mass transfer coefficient of copper ions on the cathodic side of the pellet, $c$ is the copper concentration and $f$ is the ratio of copper-deposited area to surface area of each pellet. The Faradaic current density is expressed as

$$
j_{p m}=i_{p m} n_{p}{ }^{2}=(3 / 2) \pi d^{2} f n F k c n_{p}{ }^{2}
$$

Substituting Eq. (1) into Eq. (5), one gets 


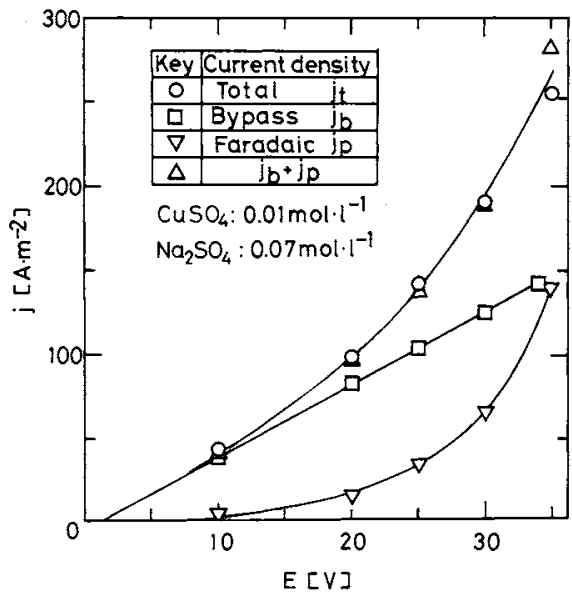

Fig. 2. Current densities via different pathways.

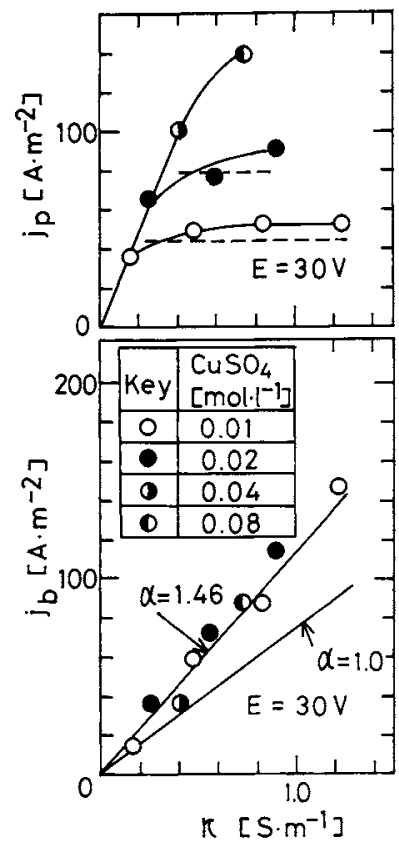

Fig. 3. Effect of electrical conductivity of electrolyte and copper concentration on current density.

$$
j_{p m}=3 \cdot 2^{1 / 3} \pi^{1 / 3} \varepsilon_{s}{ }^{2 / 3} f n F k c
$$

The ratio of copper-deposited area to surface area of each pellet was directly measured for a copper sulphate solution of $0.01 \mathrm{~mol} \cdot l^{-1}$. Figure 4 shows the value of $f$ as a function of voltage difference per pellet. The experiment of $\mathrm{Abe}^{1)}$ was carried out by using a packed bed cell described previously. ${ }^{10)}$ The value of $f$ became roughly constant $(f=0.4)$ at higher voltage differences. This means that only a part of the pellet surface is active for the mass transfer of copper ions. The mass transfer coefficient is calculated from the following equation. ${ }^{11)}$

$$
\left(1-\varepsilon_{s}\right) S h_{d}=2+0.75 R e_{d}^{1 / 2} S c^{1 / 3}
$$

The equation of Jolls and Hanratty ${ }^{7)}$ applicable to a single active particle surrounded by inert particles

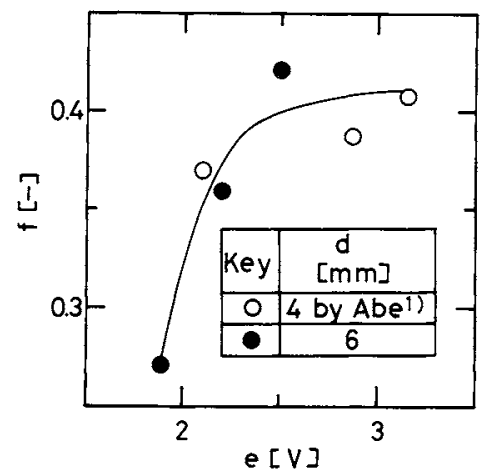

Fig. 4. Effect of voltage difference per pellet on ratio of copper-deposited area to surface area of each pellet.

gives almost the same value as Eq. (7). The Faradaic current densities calculated from Eqs. (6) and (7) are shown in Fig. 3 by the broken lines for copper concentrations of 0.01 and $0.02 \mathrm{~mol} \cdot \mathrm{l}^{-1}$, respectively. The calculated values are approximately in agreement with the experimental ones under fully polarized conditions.

Figure 5 shows the effect of gas velocity on the Faradaic current density and the bypass current density when nitrogen was bubbled into the bed. The Faradaic current density was independent of gas velocity, but the bypass current density decreased with increasing gas velocity.

\subsection{Current efficiency}

The overall current efficiency, $C_{e}$, was determined from the following equation.

$$
C_{e}=\Delta c n F / j_{t} A_{f} t
$$

where $\Delta c$ is the total amount of copper deposited in the cell and $t$ is the electrolysis period. The deposition of copper caused by the Faradaic current is expressed by

$$
\Delta c_{p}=j_{p} A_{f}\left(n_{p} L+1\right) t \eta / n F
$$

where $\eta$ is the actual current efficiency. The factor $\left(n_{p} L+1\right)$ means the number of superficial bipolar cells between the plate electrodes. Deposition of copper on the cathodic plate electrode is also caused by the bypass current.

$$
\Delta c_{b}=j_{b} A_{f} t \eta / n F
$$

The total amount of deposited copper is the sum of $\Delta c_{p}$ and $\Delta c_{b}$

$$
\Delta c=\Delta c_{p}+\Delta c_{b}=\left\{j_{p}\left(n_{p} L+1\right)+j_{b}\right\} A_{f} t \eta / n F
$$

Substituting Eq. (11) into Eq. (8), one gets

$$
C_{e}=\left\{j_{p}\left(n_{p} L+1\right)+j_{b}\right\} \eta / j_{t}=\left(j_{p} n_{p} L+j_{t}\right) \eta / j_{t}
$$

when the value of $j_{p} n_{p} L$ is higher than that of $j_{b}, C_{e}$ is nearly proportional to $j_{p} / j_{t}$.

Figure 6 shows the overall current efficiency for 


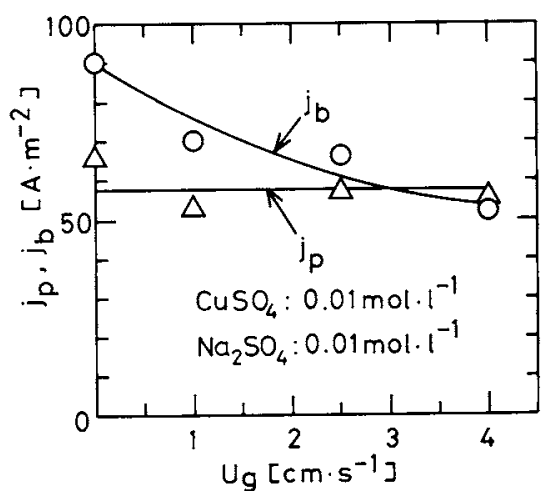

Fig. 5. Effect of gas velocity on Faradaic current density and bypass current density.

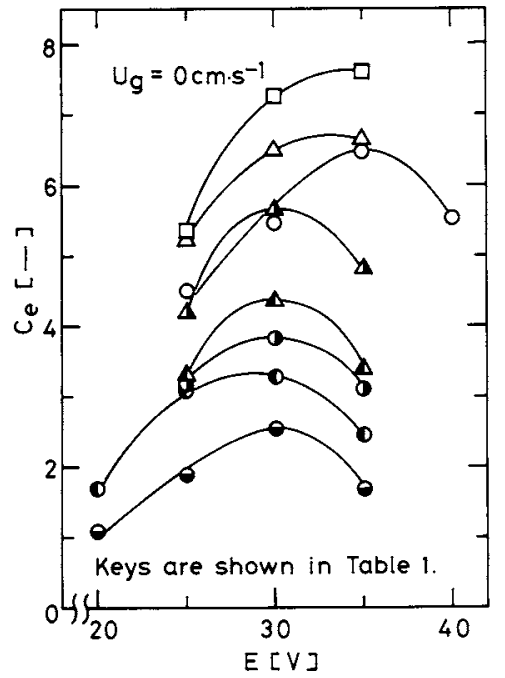

Fig. 6. Effects of electrolyte properties on overall current efficiency.

different electrolytes. The value of $C_{e}$ increased with increasing copper concentration and with decreasing electrical conductivity of the electrolyte. In the range of lower cell voltages, the overall current efficiency increased with an increase in $E$ and took a maximum at 30 to $35 \mathrm{~V}$. Beyond the optimum cell voltage, hydrogen gas was evolved and the overall current efficiency decreased.

As shown in Fig. 7, the overall current efficiency for gas-liquid systems increased with increasing gas velocity. Figure 8 shows the overall current efficiency at the optimum cell voltage. The data without nitrogen gas bubbling was well expressed by Eq. (12) with $\eta=0.52$. This low value of $\eta$ is caused by the dissolution of deposited copper with dissolved oxygen which is produced on the anodic side of the pellet. Introduction of nitrogen gas bubbles increases the maximum overall current efficiency by about $15 \%$. This is probably due to the increase in $\eta$. The displacement of dissolved oxygen by nitrogen may prevent chemical oxidation of deposited copper.

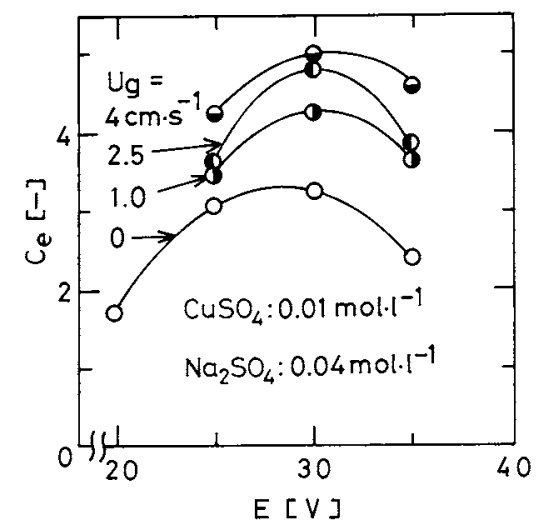

Fig. 7. Effect of gas velocity on overall current efficiency.

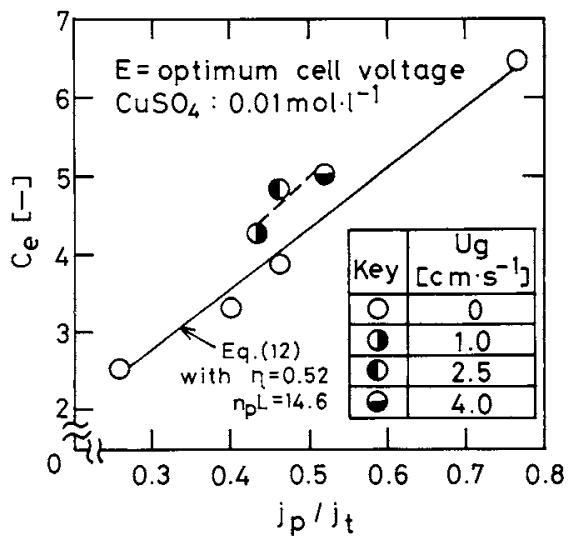

Fig. 8. Effects of $j_{p} / j_{i}$ and gas velocity on maximum overall current efficiency.

\section{Conclusion}

The bypass current density was dependent on the electrical conductivity of electrolyte and the bed configuration. When the cell constant was known, the bypass current density was calculated from Eq. (2).

The Faradaic current density under mass transfercontrolling condition was affected by the electrical conductivity and the depolarizer concentration, and was approximately expressed by Eqs. (6) and (7). The ratio of cathodic area to surface area of each electrode pellet was about 0.4 under fully polarized condition.

The overall current efficiency was expressed by Eq. (12), which was a function of the bypass current density, the Faradaic current density, the number of pellets in row, and the actual current efficiency. The overall current efficiency was improved by introducing nitrogen bubbles into the bed.

$$
\begin{array}{llr}
\text { Nomenclature } & \\
A_{f} & =\text { area of plate electrode } & {\left[\mathrm{m}^{2}\right]} \\
C_{e} & =\text { overall current efficiency } & {[-]} \\
c & =\text { copper concentration } & {\left[\mathrm{mol} \cdot \mathrm{m}^{-3}\right]} \\
\Delta c & =\text { total amount of deposited copper } & {[\mathrm{mol}]} \\
\Delta c_{b} & =\text { amount of copper deposited by bypass } & \\
& & \text { current }
\end{array}
$$




\begin{tabular}{|c|c|c|}
\hline$\Delta c_{p}$ & $\begin{array}{l}=\text { amount of copper deposited by Faradaic } \\
\text { current }\end{array}$ & $c$ \\
\hline$d$ & $=$ diameter of pellet & \\
\hline$E$ & $=$ cell voltage & \\
\hline$e$ & $=$ voltage difference per pellet & \\
\hline$F$ & $=$ Faraday constant & $\mathrm{C} \cdot \mathrm{mol}$ \\
\hline$f$ & $\begin{aligned}= & \text { ratio of copper-deposited area to surface } \\
& \text { area of each pellet }\end{aligned}$ & \\
\hline$i_{p}$ & $\begin{aligned} &= \text { Faradaic current flowing through a single } \\
& \text { pellet }\end{aligned}$ & \\
\hline$j_{b}$ & $=$ bypass current density based on $A_{f}$ & {$[\mathrm{~A} \cdot \mathrm{m}$} \\
\hline$j_{p}$ & $=$ Faradaic current density based on $A_{f}$ & {$[\mathrm{~A} \cdot \mathrm{m}$} \\
\hline$j_{t}$ & $=$ total current density & {$\left[\mathrm{A} \cdot \mathrm{m}^{-}\right.$} \\
\hline$k$ & $=$ mass transfer coefficient of copper ions & {$[\mathrm{m} \cdot \mathrm{s}]$} \\
\hline$L$ & $=$ distance between two plate electrodes & \\
\hline$n$ & $=$ number of electric charges & \\
\hline$n_{p}$ & $\begin{aligned}= & \text { number of cubic cells lined up per unit } \\
& \text { length }\end{aligned}$ & \\
\hline $\operatorname{Re}_{d}$ & $=$ Reynolds number & \\
\hline$S c$ & $=$ Schmidt number & \\
\hline$S h_{d}$ & $=$ Sherwood number & \\
\hline$t$ & $=$ electrolysis period & \\
\hline$U_{g}$ & $=$ superficial gas velocity & {$[\mathrm{m} \cdot \mathrm{s}$} \\
\hline$\alpha$ & $=$ cell constant & \\
\hline$\varepsilon_{s}$ & $=$ solid holdup & \\
\hline$\kappa$ & $=$ electrical conductivity of electrolyte & [D. 11 \\
\hline
\end{tabular}

〈Subscript〉

$m \quad=$ mass transfer-controlling

\title{
Literature Cited
}

1) Abe, S.: Bachelor thesis, Kyushu Univ. Fukuoka (1981).

2) Boussoulengas, A. V., S. Ehdaie and R. E. W. Jansson: Chemistry and Industry, 670, Oct. (1979).

3) Bruggemann, D. A. G.: Ann. Physik, 24, 636 (1935).

4) Fleischmann, M. and Z. Ibrisagic: J. Appl. Electrochem., 10, 151 (1980).

5) Goodridge, F.: Electrochim. Acta, 22, 929 (1977).
6) Goodridge, F., C. J. H. King and A. R. Wright: Electrochim. Acta, 22, 347 (1977).

7) Jolls, K. R. and T. J. Hanratty: AIChE J., 15, 199 (1969).

8) King, C. J. H. and A. R. Wright: Electrochim. Acta, 22, 1135 (1977).

9) Kusakabe, K., S. Morooka and Y. Kato: J. Chem. Eng. Japan, 14, 208 (1981).

10) Kusakabe, K., S. Morooka and Y. Kato: J. Chem. Eng. Japan, 15, 45 (1982).

11) Shirai, T.: "Ryudoso," p. 207, Kagaku Gijutsuhsa, Kanazawa (1964).

12) Yoshimura, S., A. Katagiri and S. Yoshizawa: Nippon Kagaku Kaishi, 1144 (1978).

\section{MODEL CALCULATION OF CHEMICAL REGENERATION OF SPENT CLINOPTILOLITE FROM AMMONIUM TREATMENT}

\author{
KI-SUNG HA AND MoTOYUKI SUZUKI \\ Institute of Industrial Science, University of Tokyo, Tokyo 106
}

Key Words: Ion Exchange, Adsorption, Environment, Mass Transfer

\begin{abstract}
A mathematical model to describe regeneration of spent clinoptilolite by concentrated sodium chloride solution as regenerant was developed and compared with experimental observations. The model is based on solid-phase diffusion in clinoptilolite particles and the equilibrium relation of sodium and ammonium ions. The model predicted the trend observed for both the elution curve of ammonium and the fractional regeneration of the bed.

The results of the model calculation suggest that the concentration of regenerant is one of the controlling factors in determining the regenerant volume and the fractional regeneration. Particle size is also defined as an important design parameter in determining the fractional regeneration and average eluted ammonium concentration or regenerant volume.
\end{abstract}

\section{Introduction}

There have been several papers ${ }^{2,4,6)}$ on removal of ammonium-nitrogen from wastewater by clinoptilolite in accordance with the recent stringent water

\footnotetext{
Received September 7, 1983. Correspondence concerning this article should be addressed to K.-S. Ha.
}

quality standards in water environment. The most of those papers have only reported about adsorption operation of ammonium-nitrogen from wastewater by clinoptilolite.

Design of an ammonium-nitrogen removal facility from wastewater by clinoptilolite involves the problem of regeneration of spent clinoptilolite. 